estudios del habitat FAU $=$

${ }_{155 \mathrm{~N} e 242-6483}$
Estudios del Hábitat

ISSN: 2422-6483

estudhabitat@gmail.com

Universidad Nacional de La Plata

Argentina

\title{
Norte - Sur, Este - Oeste. La conformación de la red de avenidas de la ciudad de Buenos Aires frente al avance de los automotores (1920-1940)
}

Valeria Gruschetsky**

Norte - Sur, Este - Oeste. La conformación de la red de avenidas de la ciudad de Buenos Aires frente al avance de

los automotores (1920-1940) ${ }^{1}$

Estudios del Hábitat, vol. 19, núm. 1, 2021

Universidad Nacional de La Plata, Argentina

Disponible en: https://doi.org/10.24215/24226483e096

\section{(c) (1)(0)}

Esta obra está bajo una Licencia Creative Commons Atribución-NoComercial-Compartirlgual 4.0 Internacional. 


\section{Norte - Sur, Este - Oeste. La conformación de la red de avenidas de la ciu- dad de Buenos Aires frente al avance de los automotores (1920-1940)*}

North-South, East-West. The formation of a net of avenues in the automobile era, in the city of Buenos Aires

$(1920-1940)$

Valeria Gruschetsky**

Instituto de Estudios sobre la Ciencia y la Tecnología. Universidad Nacional

DOI: https://doi.org/10.24215/24226483e096

Recepción: 6 Junio 2020

de Quilmes / Instituto de Arquitectura y Urbanismo. Universidad Nacional

Aprobación: 22 Diciembre 2020

de San Martín

Publicación: 30 Junio 2021

valeriaana@gmail.com

\section{RESUMEN:}

El avance del automóvil en la década de 1920 en la ciudad de Buenos Aires modificó de un modo relativamente veloz la vida urbana: acortó los tiempos de traslado y generó nuevas prácticas que posibilitaron vivir la experiencia de la velocidad y de la sensación de libertad a través de la circulación. Al mismo tiempo, el automóvil demandó el desarrollo de infraestructuras acordes a estas nuevas prácticas.

En este trabajo analizaremos las primeras propuestas y soluciones implementadas para posibilitar la "buena" circulación de los automotores. En estos términos, estudiaremos la conformación de la red de avenidas que cobró forma durante los años de entreguerra, focalizando en aquellos proyectos que priorizaron la funcionalidad del transporte automotor. Indagaremos sobre las intervenciones realizadas en términos de infraestructuras urbanas de movilidad. desde la acción estatal (municipal o nacional) para ver las articulaciones entre el diseño vial y el debate urbanístico.

PALABRAS CLAVES: avenidas; obra pública; Buenos Aires; infraestructuras de movilidad; automotores.

ABSTRACT: The advance of the automobile in the 1920s in the city of Buenos Aires modified urban life in a relatively fast way: it shortened travel times and generated new practices that made possible to live the speed experience and the freedom feeling through automobility. At the same time, cars demanded infrastructure development in consonance with these new practices.

The aim of this work is to analyze the first proposals and implemented solutions to enable the "good" motorist circulation. In these terms, we will study the creation of a net of avenues that took shape during the interwar years, focusing on those projects that prioritized the functionality of motoring transportation. We will examine the state policies interventions (in the local and national level) in terms of urban mobility infrastructure to see the links between motor thoroughfares' design and the urban debate.

KEYWORDS: avenues; public Works; Buenos Aires; mobility Infrastructure; automotive.

\section{Introducción}

El avance de los automotores como medio de circulación urbana, entre las décadas de 1920 y 1930, se transformó en un problema que puso en evidencia la falta de infraestructura adecuada para su desarrollo en la ciudad de Buenos Aires. El ingeniero vial Pascual Palazzo señalaba al respecto que "...la verdadera raíz del mal (...) radica, en obligar a los medios modernos de transporte a servirse de un sistema de vías medieval". Si bien se sabe que la ciudad de Buenos Aires de la Revolución Mayo y especialmente la rivadaviana se constituye a partir de la regularidad de su grilla, Palazzo al utilizar esa analogía, se podría decir un poco exagerada, intentaba dar cuenta de la existencia de calles angostas o de tipo corredor inadecuadas para el uso del transporte automotor. La voz de los expertos no era la única que participaba en este debate. La noción de "tráfico" como la dificultad de circulación por la ciudad se replicaba en la prensa desde la década de 1920. Sclabrini Ortiz en su columna del diario La Nación "A través de la ciudad" relataba lo que se demoraba transitar por la calle Corrientes antes de transformarse en avenida (La Nación, 3/8/1929), o D. Enrique Amorin escribía crónicas que tenían al "tráfico" porteño como protagonista, a la vez que reflexionaba sobre las medidas a implementar para evitar la congestión y las aglomeraciones. 
Durante la década de 1920 el crecimiento del parque automotor fue notable y la cuestión vial en las grandes ciudades se convirtió en uno de los grandes tópicos del urbanismo moderno, situación que repercutió tanto en la esfera técnica como en el ámbito de las políticas locales. En este clima de ideas, las obras de ensanches y aperturas de avenidas comenzaron a ser prioritarias en la agenda municipal de la Capital Federal, cobrando gran protagonismo en el abanico de obras públicas que realizó el Estado municipal, a las que deben sumarse aquellas otras ejecutadas por el Estado nacional en dicho territorio.

Analizar las intervenciones que conformaron el nuevo sistema de avenidas frente al avance de los automotores, vinculando el diseño vial con el debate urbanístico es el foco de atención del presente trabajo. Al mismo tiempo, intentaremos articular tales cuestiones técnicas con la dinámica política y la gestión estatal municipal y nacional. Los diferentes proyectos que dieron forma al sistema de avenidas porteñas fueron realizados por distintas reparticiones estatales que involucraron al nivel municipal -en la mayor parte de los casos- pero también al nacional. La superposición de atribuciones entre las reparticiones intervinientes brinda un marco para analizar las tensiones, las diferentes perspectivas y los conflictos que surgen en torno a una obra de infraestructura en tanto obra pública.

En este contexto surge un conjunto de preguntas que giran sobre la relación entre el tipo de avenida a realizarse y la repartición promotora o proyectista. Las variables a analizar involucran la complejidad de cada obra, los actores participantes - expertos, funcionarios públicos y políticos-, los emplazamientos, la capacidad presupuestaria de los promotores y los saberes y modelos técnicos de avenidas que primaron en cada una de las obras. Para abordar estos aspectos y las relaciones entre ellas en los distintos emprendimientos, proponemos construir un esquema a modo de plano que identifique en el espacio urbano los ensanches y las nuevas avenidas que integraron este entramado o red.

Entre las décadas de 1920 y 1930, distinguimos tres tipos de propuestas: los planes para mejorar la circulación en términos generales; los proyectos y obras que modificaron una traza existente - los ensanches de las avenidas de este a oeste- y las aperturas de nuevas vías para el tránsito - diagonales y las avenidas transversales o de conectividad norte-sur-.

La construcción de estos problemas desde una perspectiva histórica debe partir necesariamente de la profunda transformación que significó la irrupción del automóvil como medio de transporte, generando un impacto de amplio alcance que tuvo como resultado innumerables cambios tanto a nivel sociocultural como en términos político-económicos. En esta línea, el aporte de John. Urry nos brinda un marco conceptual para el análisis al entender a la movilidad como una forma de habitar. Es decir, como un movimiento espacializado a través de formas concretas, principalmente por complejas redes de infraestructuras (vías, túneles, calles, veredas, pasajes, puentes, autopistas, etc.) que han requerido planificación, inversión, y trabajo, dando forma al paisaje urbano .

Enfocados en la ciudad de Buenos Aires, debemos partir de la idea de que ha sido uno de los escenarios privilegiados de la vida política vinculada con el gobierno nacional. Esta característica condicionó su forma física; la construcción de sus edificios públicos y el desarrollo de sus infraestructuras . Estos aspectos plantean problemas vinculados con la materialización física de la ciudad que remiten a la implementación de políticas públicas y al accionar del Estado. Es decir, se trata de las obras públicas que definen a la ciudad en su condición de Capital Federal. En este sentido, la relación entre la política, la acción estatal (que incluye a los niveles nacional y municipal) y la ciudad cobra relevancia para el análisis propuesto . En este marco proponemos nuestro análisis sobre ese entramado que posibilitó la circulación y las formas de comunicación entre las diferentes áreas de la capital y sus alrededores, constituyéndose en un factor más para estudiar la expansión y densificación de la mancha urbana.

\section{Los espacios del tránsito previo al avance del automotor}

Es necesario remarcar que ese entramado de avenidas que le dio forma al sistema de circulación y movilidad de la ciudad de Buenos Aires en la década de 1930 tuvo su origen a fines del siglo XIX, cuando el primer intendente Torcuato de Alvear llevó adelante la operación que renovó el centro tradicional. 
En ese contexto, tal como señala Adrián Gorelik, se propuso la apertura de la avenida de Mayo (1888) como un "boulevard en el eje mismo de la ciudad tradicional recualificando el centro y reequilibrando el sur con el nuevo norte" junto con la proyección de un boulevard de cintura que incluía al barrio de la Recoleta pero aún muy distante de la traza del borde definitivo, la avenida General Paz. Los objetivos del proyecto eran la contención y el control por un lado y la regulación y el ordenamiento de la ciudad tradicional y su entorno inmediato por el otro $^{1}$. A fines de siglo XIX las avenidas estaban asociadas con el cambio de la imagen urbana de Buenos Aires. Alicia Novick señala que fueron un elemento clave de los procesos de modernización. En estos términos, el tránsito y la congestión de vehículos eran un aspecto más, pero no el principal problema que llevó a realización de avenidas en la ciudad. “...La negación de la cuadrícula tradicional, los modelos de las ciudades capitales, la búsqueda de espacios con perspectiva para los monumentales edificios" era lo que se buscaba con su realización . Estas también mejoraban el valor del suelo ya que permitían edificaciones con alturas mayores . La apertura de la avenida de Mayo, tal como fue proyectada, respondió a estos intereses decimonónicos. Al año de ser inaugurada, se importaba de EEUU el primer automóvil.

Los proyectos de ensanches y nuevas avenidas como parte de un proceso que llevó a mejorar las condiciones de circulación cobraron mayor relevancia recién durante las primeras décadas del siglo XX cuando se conjugaron una serie de factores. La expansión del área edificada por fuera de los límites de la ciudad tradicional puso en evidencia las dificultades de comunicación entre las diferentes zonas que comenzaron a urbanizarse tanto hacia norte como hacia el oeste. La coexistencia de vehículos de diferentes tipos de tracciones, principalmente en las calles del centro, hizo de la circulación una empresa que, con el transcurrir de los años, se fue haciendo cada vez más compleja y complicada. De esta manera, el "tráfico" comenzó a construirse como problema . La prensa fue su caja de resonancia, y la cuestión del tránsito y la congestión en la circulación, así como sus posibles soluciones se instalaron como temas nodales de la agenda municipal.

A partir de la combinación de estos factores, sumados a los aspectos que caracterizaron los modelos decimonónicos, se fueron sucediendo una serie de proyectos y obras en los que primaron la cuestión de la comunicación o de la congestión, según el caso, y que comenzaron a darle forma al entramado de arterias que conformaron el sistema de avenidas de la Capital Federal. En 1904, se elaboró un primer fundamento legal que estableció el ensanche de una serie de calles que corrían de este a oeste. Recién bajo la Intendencia de Joaquín S. de Anchorena (1910-1914) la cuestión volvió a ser considerada. Varios de los grandes proyectos urbanos del centenario se caracterizaron por la presencia de avenidas, dando lugar a una intensa controversia entre avenidas y diagonales en la planificación de la ciudad. Como resultado de este debate, se promulgaron las leyes $\mathrm{N}^{\circ} 8.854$ y Nº 8.855 en 1912 por las que se aprobó la construcción de las diagonales Roque Sáenz Peña y Julio A. Roca y la avenida 9 de Julio respectivamente $^{2}$. En rigor, como la promulgación de la ley no garantizaba en absoluto la realización de las obras, transcurrieron varias intendencias para que se llevasen a cabo.

Estas obras fueron materializadas recién entre las décadas de 1920 y 1930, mientras la congestión del tránsito y la circulación vehicular continuaban aumentando. De esta forma, la falta de infraestructura acorde a las necesidades de una ciudad que ya se había expandido hasta sus límites administrativos y se densificaba en las zonas céntricas, donde los automotores ocupaban de manera creciente los espacios destinados a la circulación, encontraron una respuesta en su puesta en marcha después de haber sido postergadas por más de una década.

\section{Una red de avenidas para Buenos Aires}

La necesidad de mejorar, readaptar o construir un sistema de avenidas y ensanchar o abrir nuevas vías que mejorasen la capacidad de circulación y las comunicaciones entre distintos puntos de la ciudad era el diagnóstico común de todo especialista o funcionario vinculado a la vialidad urbana durante las décadas de 1920 y 1930. Por supuesto, había matices, críticas y controversias entre todos los actores que 
Norte - Sur, Este - Oeste. La conformación de la Red de avenidas de la ciudad de Buenos Aires...

participaban del debate sobre cómo debería readaptarse o construirse el sistema de vías de circulación en función de los automotores dentro de un espacio urbanizado.

En este punto nos interesa adoptar el concepto de red que proviene de los estudios de movilidad para pensar el sistema de avenidas que se conformó durante los años de entreguerra en la ciudad de Buenos Aires. Jorge Blanco señala que la idea de red “...propone una perspectiva relacional para mirar la realidad, enfatizando las relaciones que se promueven entre sujetos y lugares" . Así, establece dos significados para el término, uno tecnológico y otro organizacional. Para nuestro enfoque nos resulta relevante el primero ya que entiende a las redes como un conjunto de elementos articulados entre sí, que permiten dar cuenta de las características de los sistemas técnicos del territorio. Es decir, conformar una red de avenidas implicó la fijación de infraestructuras en el territorio, que a la vez funcionaron como promotoras de diferentes procesos como fue su valorización económica y simbólica o como espacio de conformación de flujos y circulaciones diferenciadas. Las avenidas, particularmente las proyectadas a partir de la irrupción de los automotores, establecieron espacios privilegiados para experimentar la velocidad del automóvil.

Para entrar en esa discusión es necesario conocer cómo se fue conformado ese entramado de avenidas en la historia de Buenos Aires. Incluso es posible retrotraerse a las reformas emprendidas por Rivadavia en 1820, cuando surgió el primer proyecto de avenidas porteñas que prefiguraron las posteriores realizaciones. En 1827 Bernandino Rivadavia propuso que desde el boulevard Callao partiesen avenidas perpendiculares de 30 varas ( 25 metros) de ancho y cada 4 cuadras como parte de la propuesta para regularizar la ciudad y ordenar los suburbios. ${ }^{3}$ Entre ellas se encontraban los actuales trazados de las avenidas Corrientes, Córdoba, Independencia y San Juan, entre otros. Luego en 1904 se planteó la idea de ensanchar otras calles que corrían de este a oeste con la promulgación de la Ley $\mathrm{N}^{0}$ 1.583. A las ya mencionadas se les sumaba Alvear (actual avenida del Libertador entre Plaza Alvear y avenida. Dorrego) y Santa Fe hacia el norte de la avenida de Mayo/ Rivadavia y, Belgrano y Garay, hacia el sur . La realización de este tipo de obras fue lenta y despareja hasta bien entrados en la década de 1920. En muchos casos dependió de la voluntad, el interés y los recursos de cada gestión municipal. A ello se le sumaba, tal como reclamaban los técnicos, la falta de una legislación clara para llevar adelante las expropiaciones necesarias, así como también la ausencia de una normativa de edificación ajustada a las nuevas dimensiones de las avenidas ensanchadas. En esta lógica, el cambio de la línea de edificación se promulgó unos años después bajo la intendencia de Anchorena (1910-1914). Esta gestión pareció ser una excepción durante esos años ya que promulgó las leyes para las aperturas de las avenidas diagonales Norte y Sur y de la avenida Norte-Sur (9 de Julio) y, además, avanzó en algunas de las obras de los ensanches de las avenidas de este a oeste. En 1912, Santa Fe se ensanchó entre Plaza San Martín y la calle Esmeralda y Alvear desde la avenida Sarmiento a la calle Dorrego. En cuanto al resto de los ensanches, emprendió la adquisición de terrenos afectados.

Sin embargo, tal como hemos señalado, la reactivación y puesta en marcha de este tipo de obras comenzó bajo la gestión de las intendencias de Horacio Casco (5/1927 -10/1928), José Luis Cantilo $(10 / 1928$ - 9/1930) y José Guerrico (9/1930 - 2/1932) y tomaron mayor impulso recién bajo la gestión de Mariano De Vedia y Mitre (1932-1938). Los ensanches de Alvear, Santa Fe y Corrientes fueron de los primeros en realizarse en sus tramos céntricos, es decir desde la avenida Callao hacia el este. Para 1936, año del cuarto centenario de la fundación de Buenos Aires, se inauguraron muchas de estas obras como parte de los festejos que organizó la municipalidad.

La red de avenidas que comenzó a cobrar forma promediando la década de 1930 no sólo se valió de los ensanches. De las dos avenidas diagonales proyectadas en el centro en 1912, la diagonal Norte (avenida Diagonal Roque Sáenz Peña) fue la que primero se ejecutó. Partiendo desde la Plaza de Mayo, la obra avanzó lentamente e insumió más de dos décadas. Para 1926, la municipalidad la señalaba como una de sus obras más destacadas por los avances en la obtención de fondos a través de los remates de los lotes disponibles luego de las expropiaciones y demoliciones, situación que permitió su continuación .

En cuanto a la apertura de las avenidas denominadas transversales, su realización fue más compleja ya 
que se trató, en la mayoría de los casos, de obras más onerosas, a pesar de ser menos numerosas. Las dos grandes avenidas que se incorporaron a este entramado fueron la avenida 9 de Julio a fines de 1930 (proyectada en 1912 como avenida Norte y Sur, aunque el proyecto que la materializó data de 1937) y la avenida General Paz a principio de 1940 (proyectada en 1887 como avenida de circunvalación, aunque el proyecto que la materializó data 1938). Ambas implicaron grandes inversiones y se caracterizaron por ser proyectadas y ejecutadas teniendo en cuenta las necesidades de los automotores y el tránsito regional. La avenida 9 de Julio, ubicada en el centro de la ciudad, involucró la expropiación de manzanas enteras a lo largo de las treinta y tres cuadras previstas en el proyecto. La avenida General Paz en cambio, materializó la traza del límite terrestre de la ciudad, a la vez que se transformó en su acceso anular. Se trató de un proyecto que conjugó zonas con diferentes niveles de urbanización en un área donde la ciudad se mezclaba con el suburbio. Además, entre las décadas de 1920 y 1930, se proyectaron y ejecutaron otras avenidas transversales como las avenidas Costanera Sur (1926), Costanera Norte (1936) y Paseo Colón. Para esta última, promediando la década de 1930, se proyectó su remodelación, rectificación y ensanche con el objetivo de encauzar el tránsito y otorgar mayor autonomía a los vehículos rápidos. Esta avenida, recostada sobre el este la ciudad junto con su continuación, la avenida Leandro Alem (antiguo Paseo de Julio) funcionarían como punto de partida - o de llegada - de todo el conjunto de avenidas que corrían de este a oeste.

Por último, debemos mencionar una serie de nuevas avenidas o ensanches de calles que mejoraron el tránsito y las comunicaciones al oeste de lo que hemos definido como la ciudad consolidada a fines del siglo XIX - es decir, al oeste de las calles Medrano, Castro Barros y Boedo- . Entre las que corrían de este a oeste, la municipalidad destacó los trabajos realizados para ensanchar la calzada y renovar el afirmado de la avenida Cabildo como continuación de la avenida Santa Fe hasta llegar a la intersección con la avenida General Paz; el ensanche de la avenida Vértiz como continuación de la avenida Alvear (actual avenida del Liberador); la pavimentación de la avenida Centenario (actual avenida Figueroa Alcorta) hasta su empalme con la avenida Blandengues que también se pavimentó (actual avenida del Libertador). A la vez, se confeccionó el proyecto del trazado, arbolado y cruces de las avenidas Bullrich y Almeyra (antigua denominación de la avenida Juan B. Justo entre Santa Fe y Libertador) como parte de las obras del entubamiento del arroyo Maldonado ${ }^{5}$. Esta obra posibilitó la apertura de una nueva vía sobre su cauce entubado, la avenida Juan B. Justo, cuyo primer tramo fue inaugurado en 1936.

Figura 1. Plano de Buenos Aires 1936: avenidas radiales y transversales

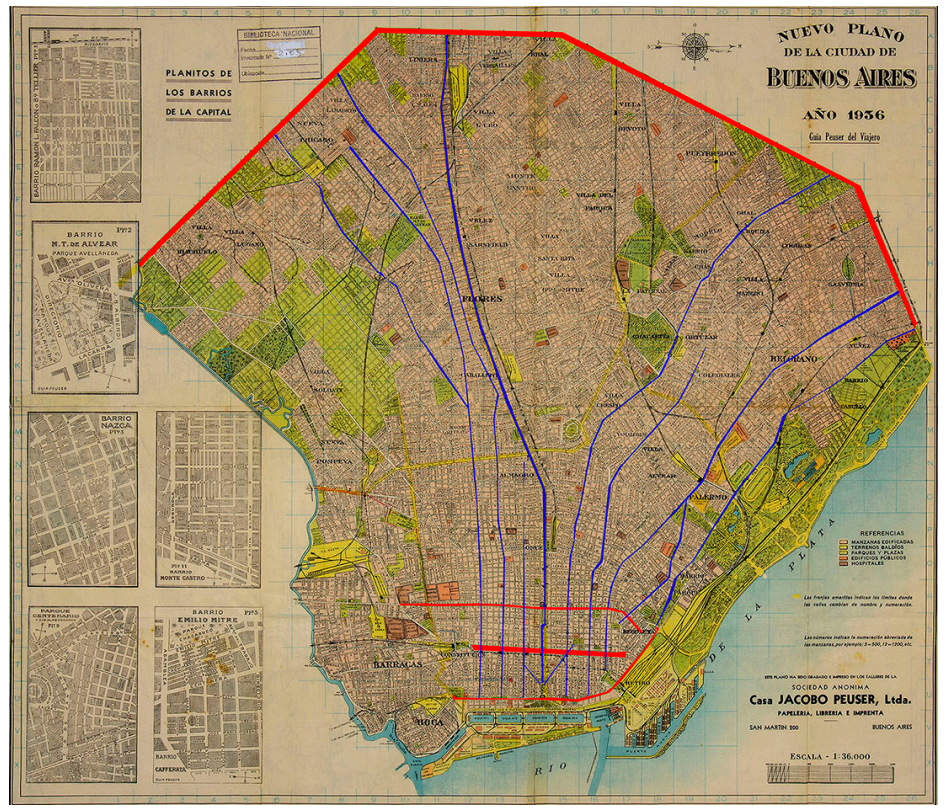

Referencias: Avenidas radiales Avenidas transversales 
Presentado este plano que permite visualizar las vías que conformaron ese entramado que configuró la red de avenidas principales de la ciudad (figura 1) ${ }^{6}$, podemos avanzar sobre las lógicas de funcionamiento que establecieron las reparticiones a cargo de los proyectos y las obras, en relación con la dinámica política y el saber profesional. El análisis se enfocará en las reparticiones y expertos que plantearon la necesidad de construir o reformar infraestructuras a partir del problema del tránsito moderno. Esta perspectiva fue la sostenida por el ingeniero Carlos María Della Paolera, director del Plan Regulador de la Municipalidad (1930-1939), quien señalaba que las disposiciones que tendían a organizar el tránsito resultaban infructuosas en el largo plazo si no eran acompañadas por proyectos de infraestructura capaces de mejorarla calidad de la circulación. ${ }^{7}$

Es decir que las propuestas tendientes a ordenar el tránsito eran, en la mayor parte de los casos, salidas parciales o de corto aliento que al contar con la ventaja de su bajo costo en términos comparativos, se transformaban en alternativas factibles a la construcción de una obra de infraestructura. ${ }^{8}$ Contra estas soluciones fáciles, muchos expertos insistían en que mejorar la red viaria y ordenar el tránsito debían ser dos factores complementarios para solucionar la congestión en la circulación. Al mismo tiempo, ponían en evidencia que solo la construcción de nuevas vías era capaz de mejorar las comunicaciones entre diferentes puntos de la ciudad y su entorno.

\section{4. ¿Quién construye las avenidas? La gestión de las obras en la ciudad}

A principios de la década de 1930, el ingeniero Jacobo Stock señalaba que para proyectar calles nuevas o ensanchar las existentes se debía cambiar la escala y tomar al automóvil como unidad. "Las calles céntricas tendrían entre 4, 6, 8 "autounidades" y no 9, 13 o 21 metros de ancho". Esta debería ser la medida para proyectar las vías adecuadas para la circulación de los automotores. Este diagnóstico construido por una mirada técnica puede considerarse compartido por los vecinos de la Capital Federal que padecían diariamente las dificultades de transitar fluidamente por las calles y avenidas céntricas. Roberto Arlt, como columnista del diario El Mundo, denunciaba las equivocaciones frecuentes del Departamento de Obras Públicas o de las empresas constructoras que provocaban la "lenta consumación” de ensanches o aperturas de calles.

\footnotetext{
Las calles Bariloche y Palma. ¿Qué diremos de Bariloche? Palma ha sido Pavimentada. Cuando terminaron de aplicarle la última mano de cemento, resultó que la calle quedaba demasiado baja. ¡Se habían equivocado! Lo que no se ha puesto en claro es a quién le cabe toda la responsabilidad de esta equivocación: si al Departamento de Obras Públicas o la empresa constructora. Se equivocaron y la calle resultó un pozo.

Se podrán argüir que equivocaciones semejantes son disculpables. Es posible; pero redundan en prejuicio de los vecinos y sirven para tirar la plata a la calle. Pero estas equivocaciones son frecuentes. El día 9 de este mes la Municipalidad mandó derribar los cordones de la vereda izquierda de la calle Corrientes, entre Uruguay y Talcahuano, porque la "línea estaba mal tirada" y había que ensanchar la vereda veinte centímetros".
}

Todavía están trabajando allí las cuadrillas de peones... (Arlt, Roberto, 1934)

Esta imagen de estado de obra permanente, particularmente, en calles con un fuerte caudal de circulación encontraba en la prensa una caja de resonancia que visibilizaba las dificultades de la gestión municipal. Esa visualización se acentuaba en el crispado clima político que culminó en el golpe de estado de 1930. Pero, vistos de otro modo, estos procesos de reforma urbana daban cuenta del impulso transformador que atravesaba a Buenos Aires en su afán de ser una "gran capital".

José Felix Uriburu, presidente de facto después del golpe de 1930, nombró a José Guerrico como intendente de la Capital Federal y disolvió al Concejo Deliberante (HCD). Este intendente conservador asignó un lugar privilegiado a la obra pública en la agenda estatal y, particularmente reactivó una serie de obras que buscaban solucionar los problemas de congestión de tránsito y mejorar las comunicaciones entre los diferentes barrios. Pero a pesar de la voluntad política y la facilidad para aunar consensos debido a que no funcionaba el HCD, la incapacidad de financiamiento repercutió en los tiempos de 
ejecución y finalización de las mismas. Recién bajo la intendencia de Mariano de Vedia y Mitre (el segundo intendente nombrado por el Presidente Agustín P. Justo -1932-1938-) la obra pública tomó el impulso necesario que posibilitó transformar y modernizar el sistema viario de toda el área metropolitana. La forma de gobernar y administrar la ciudad cambió, no sólo en términos de la activación de la obra pública, sino también en su relación con un HCD puesto nuevamente en funciones. ${ }^{9}$ La figura del intendente designada por un presidente que había sido derrotado electoralmente en la ciudad de Buenos Aires y con una victoria cuestionada en varias provincias de la República por la práctica del fraude electoral se enfrentaba a un HCD representativo de la voluntad del pueblo, expresada libremente en las urnas, y con una mayoría opositora. Esta coyuntura agudizaba la profunda contradicción que contenía en su interior el régimen municipal. ${ }^{10}$ La realización de obras públicas bajo la intendencia de De Vedia y Mitre tuvo un rol fundamental en la construcción de legitimidad no sólo frente a un HCD opositor que cuestionaba las formas de gestión municipal sino que, además, impugnaba la legitimidad política del partido gobernante.

En términos de gestión pública, la ejecución e inauguración de una gran cantidad de obras iniciadas antes de su mandato le otorgaron a la ciudad un dinamismo similar al experimentado durante la intendencia de Alvear a fines de siglo XIX. Buenos Aires dejó de ser una ciudad impotente y en estado de obra permanente, para convertirse en una ciudad en la cual se materializaba el cambio. Las obras públicas en general y las de vialidad urbana en particular le confirieron esa imagen dinámica de una ciudad moderna. La tarea de proyectar y construir el trazado, ensanche, aperturas, rectificaciones y conservación de avenidas, calles y pasajes le correspondía a Dirección de Obras Públicas de la municipalidad, pero bajo la intendencia de Guerrico se creó una nueva dependencia, la Oficina del Plan Regulador, con atribuciones mayores que contemplaban también la circulación urbana. Esta, a diferencia de la Dirección de Obras Públicas, apuntó a intervenir en la ciudad a partir del planeamiento urbano, no obstante, se generó una superposición de competencias entre ambas reparticiones en los casos de aquellas obras que implicasen cambios relevantes en la ciudad. La Oficina del Plan Regulador (devenida luego en Dirección) bajo el mando del ingeniero Della Paolera tuvo el objetivo de "racionalizar el desarrollo de la ciudad" implementando los últimos adelantos técnicos, "tanto en lo referente a comodidades como a estética", basándose en un conocimiento científico. Esta mirada integral sobre todo el espacio urbano de la Capital Federal para proyectar intervenciones relevantes, remite como antecedente a las funciones que tuvo la Comisión Estética Edilicia (CEE) que elaboró el Plano Regulador y de Reforma de la Capital Federal en 1925, aunque sus propuestas fueran desde lógicas distintas. Claramente, la nueva oficina municipal tuvo la impronta de su director quien, tal como señala Novcik, fue tributario de las ideas del urbanismo científico (donde conceptos como funcionalidad y utilidad se asociaron con la belleza y desplazaron a la mera finalidad estética) y buscó instalar la idea de planificación global a través de la realización de un plan regulador en el que primara la racionalidad .

La idea de su director era que la Oficina del Plan Regulador funcionase como un servicio técnico que ordenase y dirigiese todos los aspectos relativos a la urbanización de Buenos Aires. En estos términos, podemos interpretar que dependencias como la Dirección de Obras Públicas, con años en la gestión municipal, debiesen funcionar bajo su tutela. Un ejemplo de ello fueron las críticas que, desde el saber técnico, se realizaron sobre los ensanches y diagonales al señalar que fueron proyectadas empíricamente pero que no habían tenido en cuenta el caudal de las calles ni la densidad de la población céntrica .

En base a estos antecedentes, sumado a la labor y los conocimientos de Della Paolera, se estableció que la nueva dependencia tendría la finalidad de proveer las herramientas necesarias, principalmente a través de la recopilación y análisis de datos estadísticos, para luego elaborar proyectos parciales que solucionasen los problemas de higiene, de tráfico y de abastecimiento, así como también la construcción de barrios de acuerdo con su destino y con el carácter de su población, de espacios verdes, de vías de comunicación y del sistema de caminos de acceso. Todos estos proyectos parciales conformarían el plan de urbanización para toda la región metropolitana. Della Paolera señalaba que "la urbanización de Buenos Aires dentro de los actuales límites administrativos era una ficción dentro del organismo integral de 
la aglomeración bonaerense". Siguiendo esta línea fueron proyectadas la avenida Norte-Sur y el Paseo Colón, obras que estuvieron a cargo de la nueva oficina.

Si bien la Oficina del Plan Regulador fue ratificada con carácter definitivo cuando el HCD volvió en funciones a fines de 1932, su objetivo de asegurar el porvenir de la ciudad fijando las normas que debían regularla y su mentada autonomía del poder político, quedaron desdibujados al reducir su capacidad operativa debido a los escasos recursos que le asignaron. Novick señala que de esta forma su rol se redujo a una dependencia pequeña dentro de la estructura municipal, aunque elaboraría propuestas, investigaciones y realizaría algunas intervenciones puntuales que intentarían solucionar el problema del tráfico urbano .

En la práctica, la Dirección de Obras Públicas, a cargo del ingeniero José Estévez - un funcionario de carrera con más de veinte años en la gestión municipal-, fue la repartición que continuó con su labor en la realización de obras, teniendo muy poca interacción con la nueva dependencia, que siguió colaborando con diferentes comisiones internas de la municipalidad y entrando en contacto con algunas reparticiones técnicas nacionales. La incapacidad de imponer proyectos o soluciones técnicas a las obras bajo la tutela de la Dirección de Obras Públicas corrobora las dificultades que tuvo la Oficina del Plan Regulador en su "potencial" función de dirigir las intervenciones urbanas en la ciudad a nivel general.

La Dirección de Obras Públicas tenía mayor presupuesto, décadas de trayectoria en la gestión y fundamentalmente era una repartición de mayor relevancia en el organigrama municipal - dependía directamente del secretario de obras públicas, es decir del poder político-.${ }^{11} \mathrm{Si}$ bien fue la dependencia que estuvo a cargo de la gestión y realización de una mayor cantidad de obras, estas fueron de menor complejidad (ensanches y culminación de las avenidas diagonales). En cambio, la Oficina del Plan Regulador estuvo a cargo de la realización de la apertura de la avenida 9 de Julio, a lo que se sumó el ensanche y rectificación de la avenida Paseo Colón. A esta división de tareas debe sumarse la participación de la Dirección Nacional de Vialidad (DNV) que estuvo a cargo de la apertura de la avenida General Paz, convirtiéndose en la primera obra de vialidad urbana realizada por la repartición. ${ }^{12}$

Este esquema que asignó el diseño y ejecución de obras a diferentes reparticiones ponderando la capacidad y la experiencia de sus equipos técnicos, los intereses políticos en juego, las relaciones entre el Departamento Ejecutivo (DE) y el HCD y la capacidad de acción de las dos jurisdicciones con poder decisión sobre la ciudad, generó la atomización de las soluciones viales. Efectivamente, esta situación se profundizó cuando la obra de la apertura de la avenida General Paz pasó a manos de la DNV a fines de 1934, siendo esta una repartición dependiente del Estado nacional. Así, nuevamente, se puso en evidencia la imposibilidad de establecer una coordinación integral sobre las infraestructuras de la red de circulación de la ciudad y, menos aún, que incluyera al Gran Buenos Aires. Si bien De Vedia y Mitre contó con el apoyo del presidente Justo para la realización de buena parte de los proyectos que llevó adelante bajo su gestión, cada nivel del Estado contaba con equipos técnicos propios, que funcionaban con lógicas diferentes y priorizaban distintos intereses. Eso no significó que no hubiese vínculos entre los diferentes equipos que estaban definiendo las trazas del sistema de avenidas de Buenos Aires, pero las relaciones no eran necesariamente de interdependencia. En el caso de la obra de la avenida General Paz, la municipalidad formó parte del equipo técnico pero su papel estuvo subordinado ya que sus representantes no integraron las filas principales en donde se tomaban las decisiones. Este aspecto permite explicar la preeminencia de la lógica constructiva de los ingenieros viales por sobre la participación de los urbanistas en esta obra; situación que no se verifica en el caso de la apertura de la 9 de julio.

\section{Reformas, ensanches y aperturas: las avenidas en la era de los automotores}

\subsection{Planes y propuesta para mejorar la circulación urbana}

A partir de la publicación del Plano Regulador y de Reforma de la Capital Federal elaborado por la CEE en 1925 bajo la intendencia de Carlos Noel (1922-1927) y de los debates y reflexiones aparecidos en revis- 
Norte - Sur, Este - Oeste. La conformación de la Red de avenidas de la ciudad de Buenos Aires...

tas especializadas y en diarios como La Nación y La Prensa, cobraron forma una serie de propuestas y proyectos en los que el uso del transporte automotor comenzó a cobrar relevancia. Della Paolera, como editor de La Ingeniería en 1929, señalaba la urgencia de diseñar un sistema de avenidas para la ciudad de Buenos Aires, cuya proyección demográfica para mediados de la década de 1930 se estimaba en más de 2.000.000 de habitantes y en la que aumentarían las construcciones en altura y la cantidad de automóviles en circulación.

Dentro de este clima de ideas, especialistas en cuestiones urbanas como el ingeniero Benito Carrasco, o el grupo de expertos que integró la CEE, entre otros, elaboraron propuestas para mejorar el sistema de circulación de la ciudad en las que las aperturas de avenidas diagonales seguían teniendo gran aceptación. ${ }^{13}$ Se contemplaba mejorar o establecer fluidez en las comunicaciones entre diferentes núcleos de la ciudad que no estuviesen bien conectados entre sí, como por ejemplo Belgrano, Flores y Vélez Sarsfield. Pero, por otra parte, la lógica del diseño aplicada a través de diagonales y de "avenidas paseos" se enmarcaba en la idea del embellecimiento urbano o arte urbano, asociado a reformas practicadas en ciudades europeas entre fines de siglo XIX y la primera década del siglo XX. Es decir, los automotores y la construcción de vías de acuerdo a sus necesidades estaban presentes, pero aún no eran la variable principal que guiaba la lógica de sus propuestas.

A partir de la década de 1930, comenzaron a aparecer en Buenos Aires, al igual que en otras grandes ciudades, nuevos proyectos que contemplaban la construcción de vías a diferente nivel como forma de ordenar el tránsito y mejorar las condiciones de circulación, en especial, de los automotores. Este proceso también daba cuenta de un cambio de generación entre los arquitectos e ingenieros que participaban en los debates sobre los problemas urbanos.

Los nuevos elencos profesionales comenzaron a circular por diferentes espacios directamente vinculados a sus conocimientos específicos, entre los cuales no tardaron en aparecer, las oficinas públicas directamente relacionadas con la obra vial, tema que se integró a la práctica profesional de numerosos arquitectos e ingenieros. En este contexto, los arquitectos Ernesto Vautier y Fermín H. Bereterbide al reflexionar sobre los problemas urbanos en general, señalaban que en cuanto a las comunicaciones y la circulación era necesario organizar el tránsito y mejorar el sistema de trazados, suprimiendo los cruces entre calles a partir de la adopción de subterráneos, viaductos, puentes o autovías . El ingeniero Pascual Palazzo $^{14}$, desde la lógica de la ingeniería vial, propuso la construcción de una red de vías a bajo nivel para uso exclusivo de los automotores. Propuesta que no sólo implicaba un costo económico muy alto, sino que además significaba un importante costo en términos urbanos ${ }^{15}$.

El aumento de la velocidad de circulación para los automóviles era la variable inamovible en este tipo de proyectos. Estas ideas que ya aparecían en medios técnicos y de divulgación hacia finales de la década de 1920, cobraron mayor impulso cuando se sancionó la Ley Nacional de Vialidad a fines de 1932 y la cuestión vial cristalizó en la agenda estatal. El uso del automóvil era colocado en el centro de la escena. Las propuestas de estos expertos dejaban en evidencia la circulación de ideas y saberes donde los técnicos locales estaban al tanto de los diferentes proyectos viales que se estaban desarrollando en otras ciudades en función de mejorar las condiciones para la circulación de los automotores. Sin embargo, este tipo de propuestas no tuvieron sustento en Buenos Aires. Si bien sus costos económicos eran excesivos para el erario porteño, suponían una transformación tan grande que sugería también posibles elevados costos políticos. La destrucción de manzanas enteras en el centro y en importantes barrios de la ciudad y la necesidad de modificar las trazas de los transportes públicos, entre otros aspectos, colocaba este tipo de propuestas más en el plano de la utopía que en el de las posibilidades materiales que presentaba la ciudad de Buenos Aires a principio de la década de 1930.

\subsection{Proyectos y obras que modificaron la traza existente}

Por último, nos centraremos en aquellos proyectos que cobraron materialidad y modificaron la trama urbana. Tal como hemos explicado, gran parte de estas obras cobraron forma entre fines de la década 
de 1920 y la década de 1930 a pesar de haber sido propuestas muchos años antes. En el mejor de los casos comenzaban pero no lograban finalizarse debido a decisiones o incapacidades políticas, recortes presupuestarios o cuestionamientos de índole técnico; en otros quedaban anunciadas en normativas sancionadas pero su ejecución no llegaba a concretarse. Estos desfasajes entre la proyección y la ejecución de la obra reflejan la temporalidad propia que tiene una obra pública, sólo perceptible si se la estudia en largo plazo.

Promediando la década de 1930, comenzaron las obras para las aperturas de la avenida 9 de julio en el centro (1937) y la avenida General Paz en el límite con el Gran Buenos Aires (1938). Diseñadas como las vías urbanas de circulación rápida se transformaron simbólicamente en espacios para el uso de la velocidad que ofrecía el automóvil, para poder percibir la libertad que generaba, pero fundamentalmente, fueron presentadas como dos piezas esenciales en la construcción de una red que apuntó a mejorar la comunicación entre los diferentes puntos de la ciudad y su entorno de manera más racional.

Estas premisas fueron parte del tono modernizador que adquirió la obra pública, transformando la lógica de producción del espacio público y la cultura urbana metropolitana que caracterizo, tal como hemos mencionado, a la gestión de De Vedia y Mitre. ${ }^{16}$ A ese cambio tangible en el espacio urbano materializado con la creación de estas dos nuevas avenidas, se les debe sumar los ensanches de las avenidas céntricas realizados entre fines de la década de 1920 y la década 1930.

En cuanto a las obras de los ensanches de las avenidas que corrían de este a oeste - Santa Fe, Córdoba, Corrientes, Belgrano, Independencia, San Juan, entre otras- desde la Dirección de Obras Públicas se pretendió aumentar la capacidad de las calles, es decir mejorar el caudal y las condiciones circulatorias de los automotores, teniendo en cuenta, según el caso, a los peatones y a otros medios de transportes como los tranvías. En rigor, se trataron de obras sencillas en la mayoría de los casos, aunque existieron diferencias tanto en los costos -debido al valor de las expropiaciones a realizar- como en las características relativas a los usos y actividades que en ellas se realizaban. El problema que encontraban los técnicos y expertos más involucrados con la cuestión de la vialidad urbana en este tipo de obras era que no mejoraban necesariamente la velocidad de circulación. Bereterbide sintetizando las ideas de otros arquitectos urbanistas de su tiempo (Wladimiro Acosta y Ernesto Vautier) compartían las ideas de Palazzo que planteaban como solución construir arterias sin cruces, por consiguiente sin detenciones, aumentando de esta manera la velocidad y, en consecuencia, el caudal rodante también.

Figura 2. Ensanche de la avenida Belgrano - década de 1940

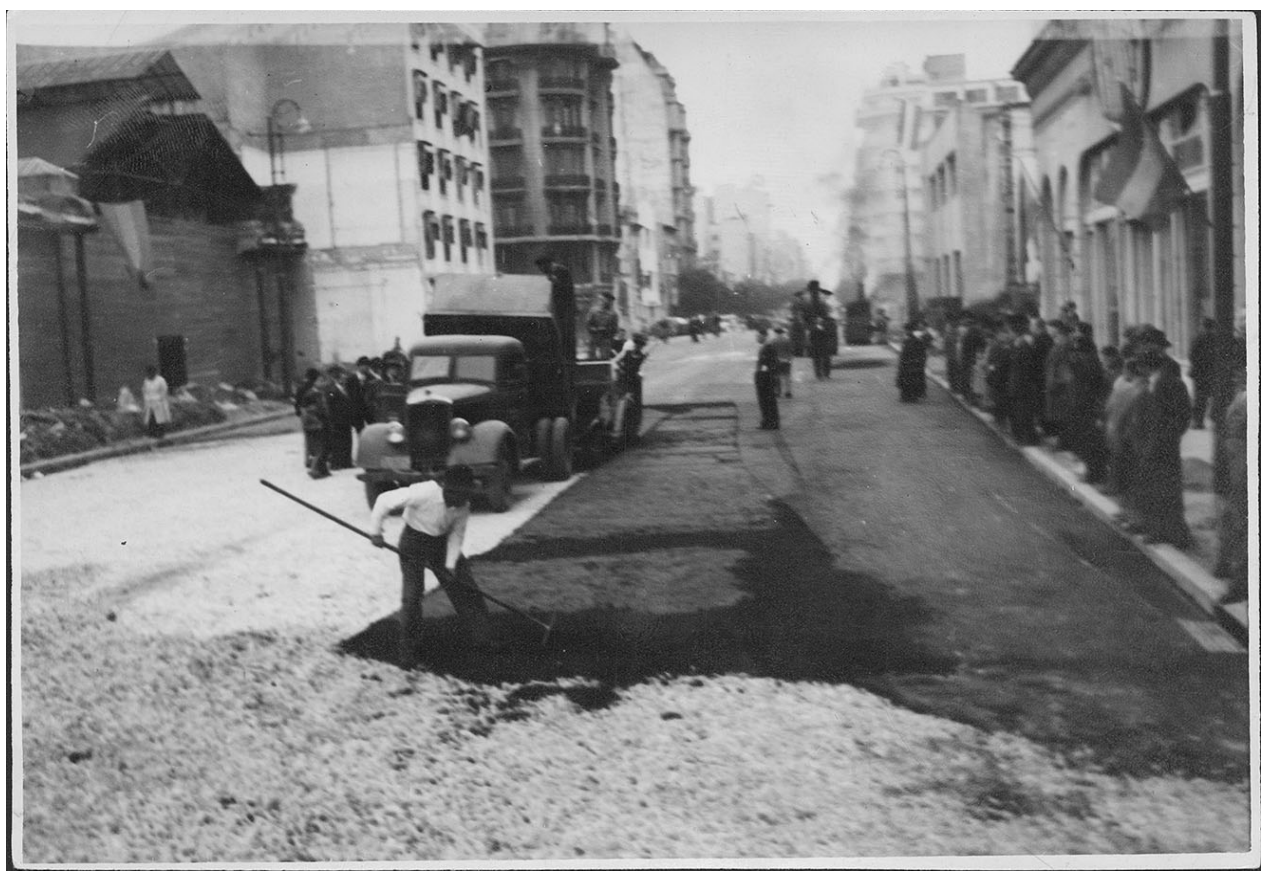

Fuente: (1940) AGN, Dpto. Doc. Fotográfico. Buenos Aires. 
Figura 3. Ensanche de la avenida Corrientes

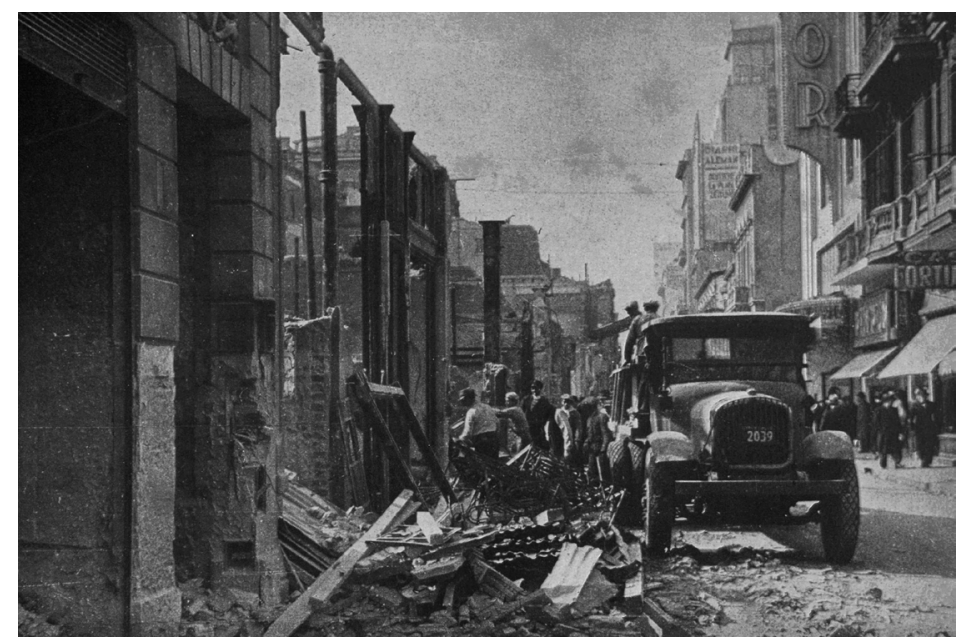

Fuente: Coppola, Horacio, Prebisch, Alberto, \& Anzoátegui, Ignacio B. (1937). Buenos Aires: Cuarto centenario de su fundación. Visión fotográfica (Municipalidad de la ciudad de Buenos Aires, Ed.; 2da.). Talleres de Editorial Atlántida.

Estas ideas se pusieron a prueba con la apertura de la avenida 9 de Julio al convertirse en esa oportunidad concreta, real y posible. Novick muestra que la multiplicidad de propuestas que se sucedieron en torno a esta avenida en la década de 1930 puede ser interpretado como un "ejercicio de estilo" de carácter proyectual en donde podemos observar la diversidad de “...ideas, referentes conceptuales, repertorios de manuales y experiencias" que se ponen en juego en un proyecto. Finalmente, en 1937 se puso en marcha el proyecto de la avenida 9 de julio, fue el director de la Oficina del Plan Regulador quien diseño y estuvo a cargo de la obra. Su primer proyecto no se encontraba demasiado distante a las propuestas precedentes, pero los costos, las posibilidades de gestión y la toma de decisiones políticas hicieron que lo modificara. En síntesis, se convirtió en una gran avenida que respondió a la necesidad de aumentar los espacios libres y verdes y solucionar los problemas del tránsito - vía de circulación y estacionamientos subterráneos- y también para la zonificación del centro de la ciudad .

En esa dirección, la obra de la avenida 9 de Julio se presentó como la alternativa que lograba condesar y resolver algunos de los grandes problemas urbanos de Buenos Aires. Sin embargo, sus críticos - los arquitectos Bereterbide, Acosta y Vautier- no tardaron en poner en cuestión esa imagen cuasi perfecta del proyecto, dando cuenta de faltas proyectuales desde el punto de vista de la circulación, la higiene y las construcciones edilicias. Además de aprovechar la circunstancia de que paralelamente se estaba construyendo la avenida General Paz (proyecto en el que participaba Vautier), otro parkway urbano pero en el límite entre la ciudad y el suburbio y así comparar ambos procesos.

Figura 4. Avenida General Paz, década de 1940

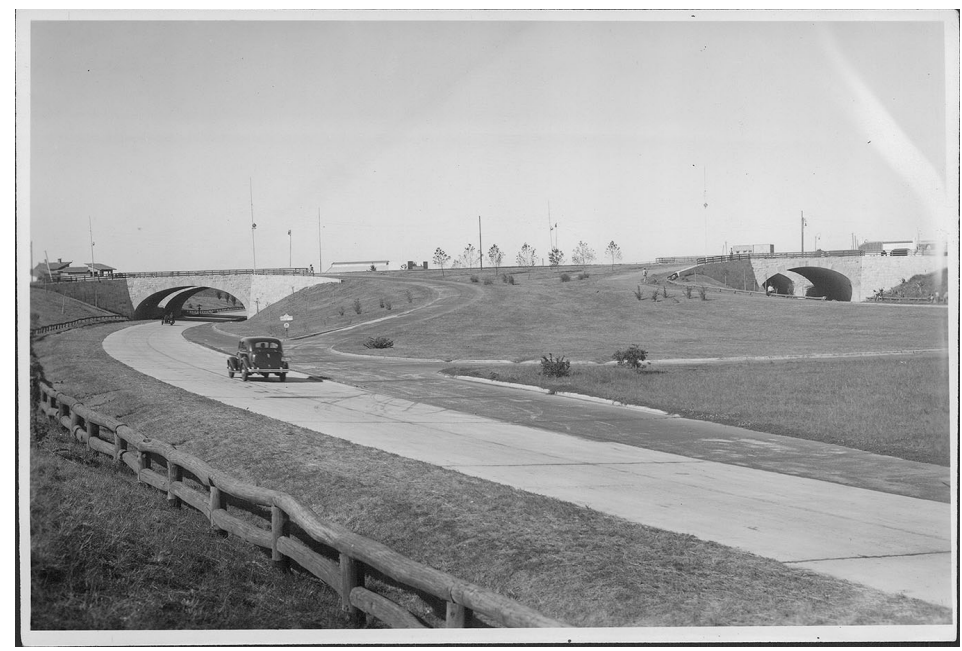

Fuente: (1943) AGN, Dpto. Doc. Fotográfico. Buenos Aires. 
Figura 5. Avenida 9 de Julio, 1946

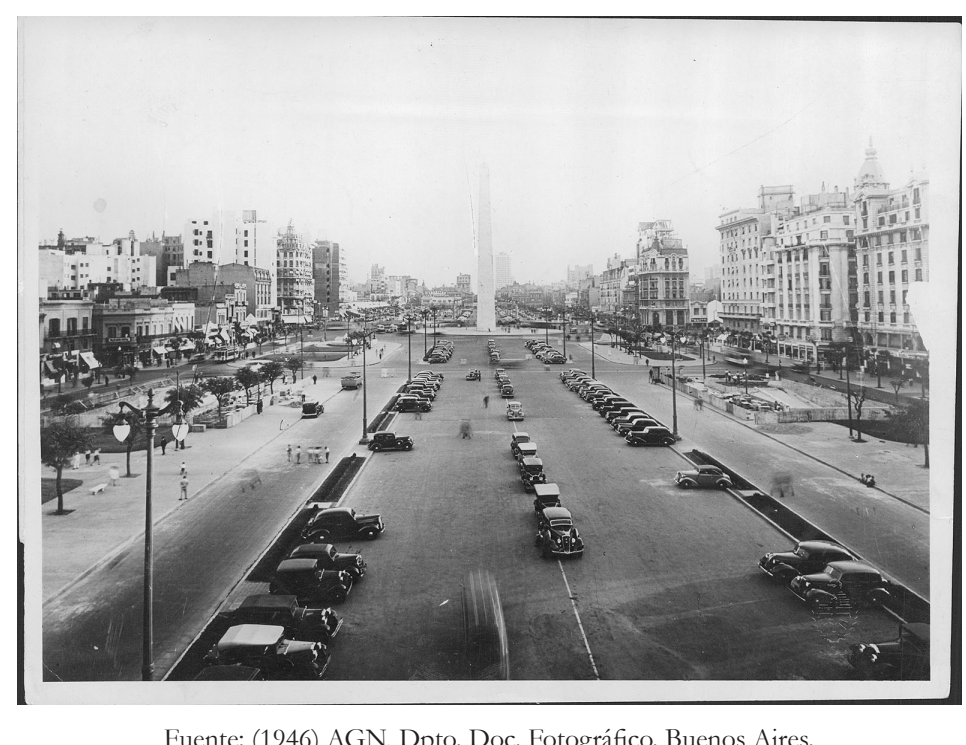

Esa controversia, por otro lado, iluminó sobre aquello que se estaba discutiendo en materia de circulación y trazados de avenidas para Buenos Aires en el ámbito profesional. Bereterbide y Acosta cuestionaban la creencia de que el tránsito en el centro predominase en dirección este a oeste y viceversa, lo que le restaba importancia a la circulación de norte a sur y viceversa. Esta situación se expandía al resto de la ciudad que al incluir a la aglomeración bonaerense se traducía en un trazado radial representado en las avenidas de este a oeste y sus continuaciones hacia el interior y suburbios. En estos términos, Buenos Aires carecía de un sistema de avenidas de cintura que relacionase transversalmente entre sí a los distintos núcleos de la ciudad. Las excepciones, según los arquitectos, eran la avenida General Paz cuya obra recién había comenzado- y las avenidas Callao- Entre Ríos y Alem- Colón, manifiestamente insuficientes, estas últimas, para el progresivo movimiento entre el norte y el sur de la Capital. A este esquema se le sumaba la avenida 9 de Julio.

\section{Palabras finales}

Estas múltiples miradas, que en términos de propuestas de infraestructuras de movilidad urbana no parecen tan encontradas, pusieron de manifiesto otro tipo de cuestiones que cobran relevancia a la hora de analizar la relación entre los proyectos que despertaron mayor "entusiasmo" entre urbanistas, arquitectos e ingenieros y aquellos otros que, sin perder trascendencia, fueron realizados por los técnicos de las reparticiones a cargo. Las reflexiones y debates sobre las obras en particular o sobre la red de avenidas en general refuerzan el significado que tuvo la oportunidad de encontrar un espacio en la ciudad para proyectar y crear avenidas ajustadas a las necesidades del automóvil, así como también para repensar la comunicación vial de la Capital Federal y su entorno.

Finalmente, esa oportunidad cristalizó y se materializó en esas dos nuevas avenidas transversales que integraron la red de avenidas porteñas entre fines de la década de 1930 y principios de la década de 1940. La avenida 9 de Julio (aunque sólo fueran cinco de las treinta y tres cuadras proyectadas) se inauguró en octubre de 1937 y la avenida General Paz en Julio de 1941. Pero además, estas obras se tradujeron en posibilidades de acción y visibilidad para las dos reparticiones que las llevaron a cabo. Dos reparticiones nuevas en la estructura estatal, una en el ámbito nacional y la otra en el municipal, que desde la composición de sus cuerpos técnicos - profesionales y expertos - y a partir de una dinámica de gestión marcada por la eficiencia y la racionalidad también buscaron representar ese tono modernizador que le interesaba visibilizar a la gestión estatal de la década de 1930 y que se plasmó en las obras, por lo menos simbólicamente. 


\section{BIBLIOGRAFÍA}

Aliata, F. (2006). La ciudad regular: Arquitectura, programas e instituciones en el Buenos Aires posrevolucionario, 1821-1835. Prometeo 3010

Amorin, E. M. (1927). Tráfico. Buenos Aires y sus aspectos. Editorial Latina.

Arlt, R. (1934, abril 11). Aguafuertes Porteñas. Fantasías Municipales en Liniers. El Mundo.

Ballent, A. (2016). El Estado como problema: El Ministerio de Obra Públicas y el centro de Buenos Aires durante la presidencia de Agustín P. Justo, 1932-1938. Estudios del Hábitat, 14(2), https://revistas.unlp.edu.ar/Habitat/article/view/e005

Bereterbide, F. H., \& Vautier, E. E. (1933). ¿Qué es el urbanismo. Concejo Deliberante de la Municipalidad de Buenos Aires.

Bereterbide, F. H. (1932). Proyecto de ejecución de la avenida transversal del Norte a Sud. Revista de Arquitectura, 141, 408-421.

Bereterbide, F. H., \& Acosta, W. (1937). Aspectos higiénicos y de circulación de la Avenida Norte-Sud. Nuestra Arquitectura, 99, 343-353.

Blanco, J.. (2018). Redes. En D. Zunino Singh, G. Giucci, \& P. Jirón (Eds.), Términos clave para los estudios de movilidad en América Latina (pp. 153-159). Editorial Biblos.

Bonicatto, V. (2011). Escribir en el cielo. Relatos sobre los primeros rascacielos en Buenos Aires (1907-1929) [Tesis de Maestría. Universidad Torcuato Di Tella].

Carrasco, B. J. (1927). Algunas consideraciones sobre la urbanización de las ciudades. Recopilación de artículos publicados durante los años 1923 al 26. Los amigos de la ciudad.

Comisión de Estética Edilicia. Intendencia Municipal. (1925). Proyecto orgánico para la urbanización del Municipio. El plano regulador y de reforma de la Capital Federal. Talleres Peuser.

Coppola, H. Prebisch, A, \& Anzoátegui, I. B. (1937). Buenos Aires: Cuarto centenario de su fundación. Visión fotográfica (Municipalidad de la ciudad de Buenos Aires). Ed.. 2da.. Talleres de Editorial Atlántida.

Del Mazo, M. (1931). La congestión del tráfico. Su solución inmediata en la Capital Federal. Ferrati Hnos.

Della Paolera, C. M. (1929). Urbanismo y problemas urbanos de Buenos Aires. La Ingeniería, 660, 440-456.

Editorial. (1932). Del plan regulador del desarrollo y extensión de la Ciudad de Buenos Aires. Revista de Arquitectura, 136, 158-159.

Revista Municipal, (1904, Julio y Septiembre).

Gorelik, A. (1998). La grilla y el parque: Espacio público y cultura urbana en Buenos Aires, 1887-1936. Prometeo /Universidad Nacional de Quilmes.

Gruschetsky, V. (2008). El espiritu de la calle Corrientes no cambiará con el ensanche”. La transformación de la calle Corrientes en avenida. Debates y representaciones. Buenos Aires 1927-1936 [Tesis de licenciatura inédita, Universidad de Buenos Aires].

Gruschetsky, V. (2018). La avenida General Paz. Infraestructuras de movilidad urbana, expertos y política en Buenos Aires (1887-1941). [Tesis de doctorado inédita, Universidad Torcuato Di Tella].

Gruschetsky, V. (2019). La Dirección Nacional de Vialidad, una repartición modelo del Estado Nacional. Técnica y política en la Ciudad de Buenos Aires en la década de 1930. En: L. Menazzi \& G. Jajamovich (Eds.), Saberes urbanos (pp. 77-111). Teseo.

Gruschetsky, V. (2020). Nuevas avenidas, nuevos paisajes. La transformación de Buenos Aires a través de las infraestructuras de movilidad durante la década de 1930. Coordenadas. Revista de Historia Local y Regional, VII (2), (pp 121-145). 
Norte - Sur, Este - Oeste. La conformación de la red de aVenidas de la ciudad de Buenos Aires...

Memoria Municipal de la Ciudad de Bs. As, (1912, 1926, 1933-1934, 1935, 1936).

Novick, A. (2004). Della Paolera. En J. F. Liernur \& F. Aliata (Eds.), Diccionario de Arquitectura en la Argentina. Estilos, obras, biografía, instituciones, ciudades (pp. 192-193). AGE.

Novick, A. (2004). Della Paolera. (2011). La avenida más ancha del mundo. Política, arquitectura e imagen. Anales del Instituto de Arte Americano e Investigaciones Estéticas, 41, 133-154.

Novick, A., \& Piccioni, R. E. (2004). Avenida. En J. F. Liernur \& F. Aliata (Eds.), Diccionario de Arquitectura en la Argentina. Estilos, obras, biografía, instituciones, ciudades (pp. 98-102). AGE.

Palazzo, P. (1933). El tráfico en Buenos Aires. Red de vías a bajo nivel. Solución de problemas. Talleres gráfico A. Biocco y Cia.

Paterson, M. (2007). Automobile politics: Ecology and cultural political economy. Cambridge University Press.

Peuser, J. (1936). Nuevo Plano de la Ciudad de Buenos Aires 1936. Guía Peuser del viajero [Map]. Sociedad Anónima Jacobo Peuser, LDA.

Privitellio, L. de. (2003). Vecinos y ciudadanos: Política y sociedad en la Buenos Aires de entreguerras. Siglo Veintiuno editores.

Stock, Jacobo. (1932,). El problema del tráfico en Buenos Aires. Revista de Arquitectura, 191.

Urry, J. (2007). Mobilities. Polity Press.

Vautier, E. E. (1933, julio). La reglamentación de las construcciones en la avenida Norte-Sud. Revista de Arquitectura, Junio.

Walter, R. J. (1993). Politics and urban growth in Buenos Aires, 1910-1942. Cambridge University Press.

Wollen, P., \& Kerr, J. (2002). Autopia: Cars and culture. Reaktion Books.

Zunino Singh, D., Giucci, G., \& Jirón, P. (2018). Términos clave para los estudios de movilidad en América Latina. Editorial Biblos. 


\section{NOTAS}

${ }^{*}$ Este trabajo se basa en versiones preliminares que han sido discutidas en congresos y jornadas académicas. Los comentarios de Melina Piglia y Dhan Zunino Singh (XVI Jornadas Interescuelas / Departamentos de Historia. Mar del Plata, 2017 - presentado en actas "El sistema de avenidas de la ciudad de Buenos Aires en la era de los automotores (1920-1940)" fueron de suma importancia para la elaboración de la versión final del mismo. Finalmente, debo aclarar que el artículo se desprende de mi tesis de doctorado inédita defendida en octubre de 2018, más específicamente toma como base el capítulo 3 en donde abordo los problemas de circulación de automotores en la ciudad y la conformación de la red de avenidas en tanto infraestructuras urbanas.

** Doctora en Historia (UTDT), Licenciada en Historia y Profesora de Enseñanza Media y Superior en Historia (UBA). Es docente universitaria y de posgrado en CBC y FADU-UBA; IA-UNSAM y UTDT. Es miembro del Laboratorio Espacio, Tecnología y Cultura IESCT- UNQ. Sus temáticas de estudio son la historia urbana y de la ciudad focalizando en las infraestructuras de movilidad urbana y en las políticas públicas y burocracias estatales y ha publicado artículos en libros y revistas académicas nacionales e internacionales, entre otros medios.

\footnotetext{
${ }^{1}$ Sobre la transformación que llevó adelante el Intendente Torcuato de Alverar y sobre las diferentes propuestas que existieron para construir un boulevard de circunvalación para la ciudad de Buenos Aires.

${ }^{2}$ Para los debates en el Concejo Deliberante y la tensión entre la realización de las obras y los tiempos de la política.

${ }^{3}$ Fernando Aliata ha trabajado sobre las propuestas de renovación y regulación - en el trazado y en la nivelación del suelo- de la ciudad, donde una de sus premisas fue la rectificar las calles para mejorar la circulación (además del control censal y electoral) en función de generar las posibilidades de expansión.
}

${ }^{4}$ Tal como señala Alicia Novick su inauguración el 12 de octubre de 1937 significó la apertura de sólo 5 cuadras de las treinta y tres previstas. Las obras siguieron avanzando hasta 1940 cuando se detienen por falta de transparencia en el proyecto y recién serán retomadas en la década de 1960 .

${ }^{5}$ El primer tramo del arroyo Maldonado se entubo entre 1929-1933 -5 km hasta la calle Bolivia-, el segundo finalizó en 1939 - hasta la calle Segurola- y en la década de 1940 se llegó hasta la Avenida General Paz (información reconstruida en base al acceso a diferentes páginas de internet y de la Memorias Municipales de la ciudad de Buenos Aires)

${ }^{6}$ En el plano de Buenos Aires resaltamos las avenidas radiales (se trataron, en su gran mayoría, de obras de ensanches de calles existentes) y avenidas transversales (se trataron, en su mayoría de aperturas de nuevas vías).

${ }^{7}$ Carlos María Della Paolera además de ingeniero era urbanista.

${ }^{8}$ Un ejemplo de las políticas de ordenamiento del tránsito se observan en varios de los escritos de Marcelino del Mazo, un especialista sobre cuestiones de vialidad urbana, que a principios de los años treinta recuperaba un dictamen de la Comisión Asesora de Tráfico del Concejo Deliberante de 1927 para dar cuenta de la relevancia de este tipo de políticas las políticas de ordenamiento del tránsito.

${ }^{9}$ Richard Walter señala que en muchas ocasiones los frecuentes enfrentamientos entre ambos poderes se tradujeron en acciones por parte del poder ejecutivo que ignoraron o sobrepasaron al poder legislativo, pues aquel puso en marcha medidas y proyectos por su propia cuenta, dejando a un HCD impotente y frustrado por los resultados de los debates y protestas.

${ }^{10}$ Con la sanción de la nueva Ley electoral municipal de 1918, el presidente H. Yrigoyen se opuso a que el intendente de la Capital Federal fuese elegido mediante la práctica del sufragio universal, al advertir que el control de municipio era clave para el ejercicio del poder político ya que se trataba de una jurisdicción particular al ser también la sede político-administrativa del poder político nacional.

${ }^{11}$ Alicia Novick da cuenta de esta rivalidad y/o competencia en los técnicos de las dos reparticiones en relación a la apertura de la avenida 9 de Julio . Por otro lado, al observar la disposición de las memorias municipales - orden de aparición y cantidad de páginas- también podemos dar cuenta de la relevancia de cada repartición dentro de la gestión de la municipalidad. (Memoria Municipal de la Ciudad de Buenos Aires, 1933-1934;1935 y 1936).

${ }^{12}$ En 1932 se sancionó la Ley 11.658 (Ley Nacional de Vialidad) que dio origen a la Dirección Nacional de Vialidad que tuvo la función de crear un sistema troncal de camino nacionales de bajo costo que uniese las capitales de provincias, los puertos y las estaciones de ferrocarril. Con las obras de la avenida General Paz y el puente Nicolás Avellaneda sobre el Riachuelo el espacio urbano comenzó a ser intervenido por la repartición.

13 Mientras Carrasco se inspiraba en el movimiento City Beatiful y más precisamente en uno de sus proyectos emblemáticos: el plan radial o de abanico de Daniel Burham para la ciudad de Chicago en 1909, la CEE lo reinterpretaba y combinada del sistema monumental 
Norte - Sur, Este - Oeste. La conformación de la red de avenidas de la ciudad de Buenos Aires...

francés encuadrado en las intervenciones del Baron d' Haussmann, con el concepto más reciente de los trazados irregulares y asimétricos sostenidos por los urbanistas ingleses y alemanes y aplicados en los planos de Joseph Stubben, Camilo Sitte y otros...", ideas estaban desarrolladas en función de vehículos a tracción a sangre.

${ }^{14}$ Futuro director del proyecto de la apertura de la avenida General Paz, (1937-1941).

${ }^{15}$ Su proyecto era similar o tomaba como referencia al plan pensado para la ciudad de Nueva York The Regional of New York and Environs de 1929. Este plan elaborado por los ingenieros Nelson Lewis y Harold Lewis fue la base conceptual para muchos de los parways que Robert Moses construyera durante los años de 1930 y de 1940 como funcionario público del área metropolitana de Nueva York. (Owen D. Gutfrund, 2007).

${ }^{16}$ Gorelik sintetiza este proceso que caracterizo a la gestión municipal de Mariano De Vedia y Mitre como "una nueva coloración, que sobreimprime al clima reformista (de la década de 1920) en un novedoso clima excluyentemente modernizador.” (Gorelik, 1998, p. 391). 